\title{
HIGH-FIDELITY SIMULATIONS OF A STANDING-WAVE THERMOACOUSTIC-PIEZOELECTRIC ENGINE
}

\author{
J. Lin ${ }^{1 *}$, C. Scalo ${ }^{2}$, and L. Hesselink ${ }^{1}$ \\ ${ }^{1}$ Department of Electrical Engineering, Stanford University, Stanford, CA 94305, USA. \\ ${ }^{*}$ Corresponding author's e-mail: linjef@stanford.edu \\ 2 Department of Mechanical Engineering, Purdue University, West Lafayette, IN 47907, USA.
}

Keywords: thermoacoustics, impedance, piezoelectric, energy conversion, energy extraction, NavierStokes, standing-wave
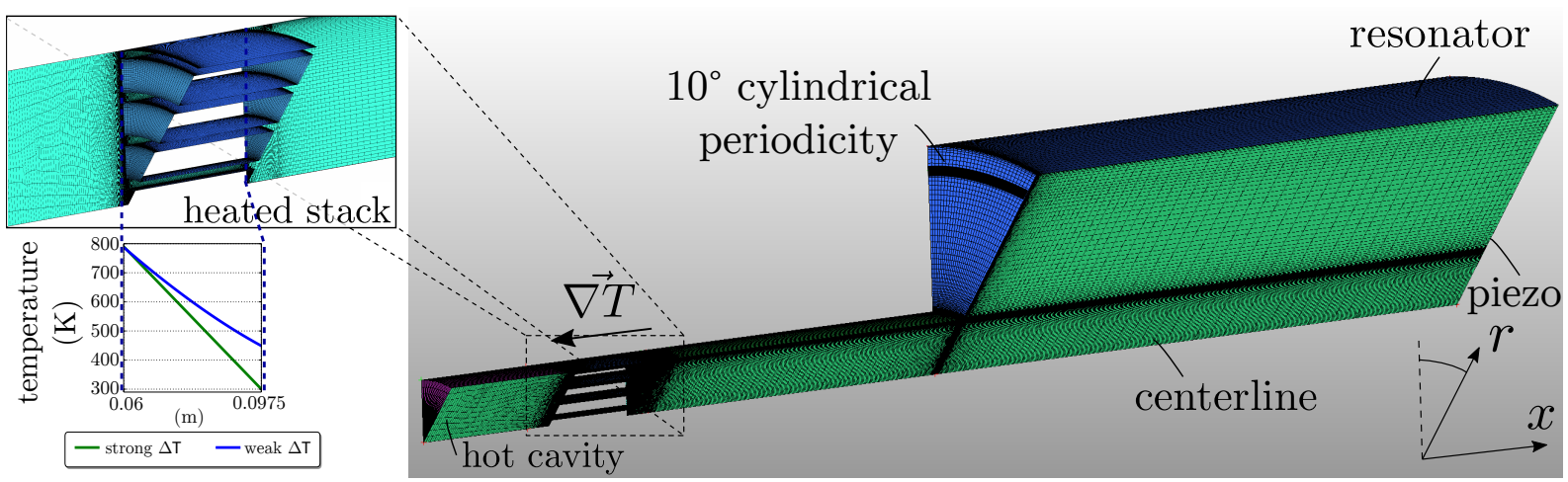

Figure 1: Computational grid meshing of a $10^{\circ}$ wedge of the axially-symmetric engine geometry. Linear temperature gradient profiles are introduced in the heated stack.

We have carried out fully compressible Navier-Stokes numerical simulations of a standingwave thermoacoustic piezoelectric (TAP) engine model inspired by the experiments of Smoker et al. (2012) [2]. The TAP model is composed of a $51 \mathrm{~cm}$ long cylindrical resonator divided into two constant-area segments of $19.5 \mathrm{~mm}$ and $71 \mathrm{~mm}$ in diameter. The smaller diameter segments contain a thermoacoustic stack while the larger diameter segment is capped by a PZT-5A piezoelectric diaphragm. The diaphragm is tuned to the engine's natural operating frequency $(388 \mathrm{~Hz})$ for maximization of acoustic energy extraction.

The adopted numerical setup allows for both the evaluation of the nonlinear effects of scaling and the effect of a fully electromechanically-coupled impedance boundary condition, representative of a piezoelectric element. No signs of acoustic nonlinearities are present. Building upon the computational platform as presented by Scalo et al. (2015) [1], the stack geometry is now fully resolved without the use of source terms representing drag and heat transfer. Geometry meshing and example temperature profiles are shown in figure 1. To our knowledge, fully broadband-matching impedances have not been coupled with Navier-Stokes thermoacoustic engine simulations, and prior research has been performed on this engine using models such as the impedance model in DELTAEC.

We have performed simulations without the piezoelectric diaphragm with different temperature gradients across the stack, including values at $\Delta T=490 \mathrm{~K}$ and $\Delta T=340 \mathrm{~K}$, which respectively reach limit cycles at cavity amplitudes of $P_{\lim }=356 \mathrm{~Pa}$ and $P_{\lim }=6109 \mathrm{~Pa}$. Intermediate values of $\Delta T$ were also investigated and growth rates in the linear regime were validated with linear stability analysis of the engine.

To construct an energy extraction boundary condition, we imposed a time-domain impedance 


\section{frequency response}

$$
\left[\begin{array}{c}
\hat{u} \\
\hat{I}
\end{array}\right]=\left[\begin{array}{ll}
Y_{11}(\omega) & Y_{12}(\omega) \\
Y_{21}(\omega) & Y_{22}(\omega)
\end{array}\right]\left[\begin{array}{c}
\hat{p} \\
\hat{V}
\end{array}\right]
$$

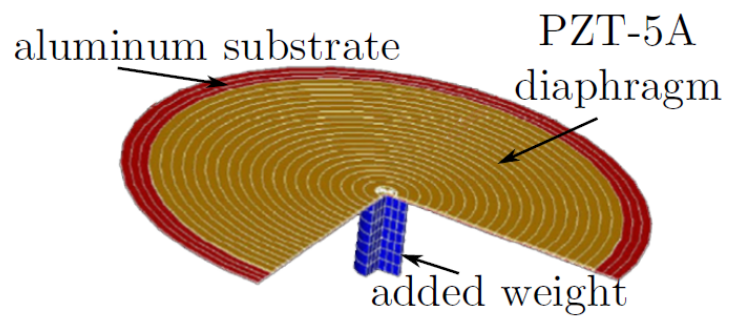

$$
\begin{aligned}
& u \triangleq \operatorname{velocity}[\mathrm{m} / \mathrm{s}] \quad p \triangleq \operatorname{pressure}[\mathrm{Pa}] \\
& I \triangleq \operatorname{current}[\mathrm{A}]
\end{aligned}
$$

Figure 2: The piezoelectric diaphragm used by Smoker et al. was tuned and weighted to the engine operating frequency and was reported as a two-port model with associated transfer functions $Y$. In order to construct computationally-compatible impedances, the two-port model was reduced to a one-port model of the form $\hat{p}=Z(\omega) \hat{v}$.

boundary condition as a representation of the piezoelectric diaphragm as shown in figure 2 . We have collapsed the two-port model provided by the PZT-5A piezoelectric patch by using the reference resistance of $3170 \Omega$ and constructed both single-frequency and broadband acoustic, causal impedances, as shown in figure 3. These impedance boundary conditions were coupled with the Navier-Stokes solver, producing both a high-fidelity simulation of the engine and simulation results for acoustic-to-electric energy conversion.
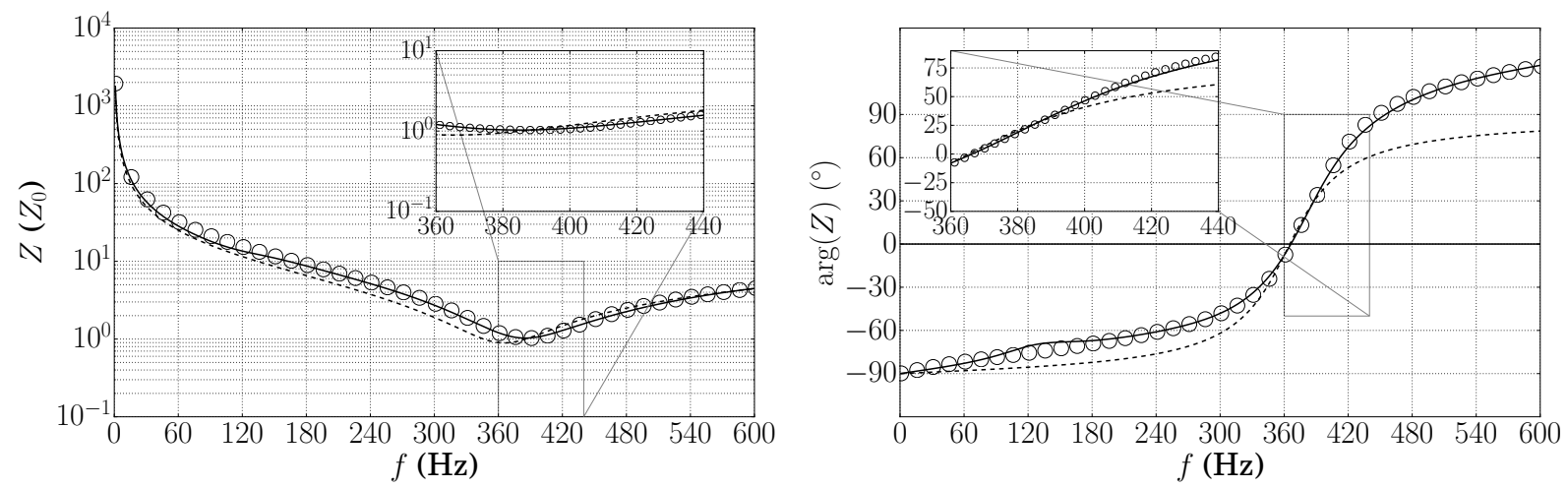

Figure 3: Broadband fitting of a set of Helmholtz pairs and fitting of a Helmholtz pair to the transmittance-matrix derived impedance; measured impedance from experimental data collapsed into one-port model (o ० o), single-frequency Helmholtz oscillator (fitted around $388 \mathrm{~Hz})(----)$, and broadband impedance using multiple basis frequencies (- ). Fitting around the engine operating frequency is shown in the inset, demonstrating a reasonable ability for single-pole impedances to be sufficient for monotonic simulations.

Results at a low-amplitude regime were validated against results obtained from experimental results in literature. Energy extraction results are within 10\% difference.

\section{References}

[1] Scalo, C., Lele, S. K., and Hesselink, L. "Linear and nonlinear modeling of a theoretical traveling-wave thermoacoustic heat engine". 766, , 368-404.

[2] Smoker, J., Nouh, M., Aldraihem, O., and Baz, A. "Energy harvesting from a standing wave thermoacousticpiezoelectric resonator". Journal of Applied Physics, 111, (2012), 104901. 